Submitted for publication in Nuclear Intruments and Methods, B, proceedings of the $16^{\text {th }}$ Internat'l Conference on Ion Beam Analysis, Albuquerque, NM, June 29-July 4, 2003

\title{
Open questions in electronic sputtering of solids by slow highly charged ions with respect to applications in single ion implantation
}

\author{
T. Schenkel ${ }^{1, \#}$, I. W. Rangelow ${ }^{*}, 1$, R. Keller ${ }^{1}$, S. J. Park ${ }^{1}$, J. Nilsson ${ }^{2}$, A. Persaud ${ }^{1}$, V. R. \\ Radmilivitc $^{1}$, J. A. Liddle ${ }^{1}$, P. Grabiec ${ }^{* *}, 1$, J. Bokor ${ }^{3}$, and D. H. Schneider ${ }^{2}$ \\ ${ }^{1}$ E. O. Lawrence Berkeley National Laboratory, Berkeley, CA \\ ${ }^{2}$ Lawrence Livermore National Laboratory, Livermore, CA \\ ${ }^{3}$ Department of Electrical Engineering and Computer Science, University of California, \\ Berkeley, CA
}

In this article we discuss open questions in electronic sputtering of solids by slow, highly charged ions in the context of their application in a single ion implantation scheme. High yields of secondary electrons emitted when highly charged dopant ions impinge on silicon wafers allow for formation of non-Poissonian implant structures such as single atom arrays. Control of high spatial resolution and implant alignment require the use of nanometer scale apertures. We discuss electronic sputtering issues on mask lifetimes, and damage to silicon wafers.

\# Email: T_Schenkel@LBL.gov 


\section{Introduction}

The development of ECR and EBIT ion sources in the 80ies has made beams of relatively slow $\left(\mathrm{v}<\mathrm{v}_{0}\right)$, highly charged ions (SHCI) available for ion solid interaction studies $[1,2]$, and a wide array of new collision phenomena has since been quantified where the interaction of ions with solids is dominated by the deposition of potential energy of highly charged ions. SHCI like $\mathrm{Xe}^{44+}$ or $\mathrm{Au}^{69+}$ relax into charge state equilibrium within a time of only about $10 \mathrm{fs}$ following penetration of a solid. Dielectronic processes, i. e. autoionization and Auger transitions, in a transient hollow atom mediate this rapid relaxation. Due to the swift relaxation, deposition of potential energy occurs close to the sample surface. The deposition of potential energy is associated with intense electronic excitation of the surface near target volume and increased removal of material has been reported for several dielectrics (oxides, and alkali halides) as well as for GaAs for very high ion charge states $[3,4]$. Irrespective of the details of hollow atom formation and decay, which pose many intriguing basic atomic physics questions, SHCI essentially form large numbers of hot electrons in solids. These electrons excite target atoms and molecules, and electron emission into the vacuum can leave a nano-scale volume charged. Mechanisms of electronic sputtering by SHCI are similar to those in swift $(>1 \mathrm{MeV} / \mathrm{u})$ heavy ion interactions with solids in that they are very materials specific (e. g., defect mediated though compound specific formation and decay of self trapped excitons or holes) and subject of ongoing controversial debates (e. g., thermal spike vs. Coulomb explosion). Recent reviews of the state of these debates can be found in $\operatorname{Ref}[3,4]$. In this article we discuss issues of electronic sputtering by highly charged dopant ions in the context of our development of a single ion implantation technique. 


\section{Experimental results and open questions}

In single ion implantation, single ion detection with effectively $100 \%$ detection efficiency can be achieved when high secondary electron emission yields form SHCI are used for ion impact registration [5]. The electron emission is largely independent of the kinetic energy of SHCI, and increases for very low impact energies due to enhanced time for above surface relaxation [2]. Very low impact energies translate into shallow range profiles with minimal straggling. This becomes important in the formation of single atom devices with single dopant placements requirements below $+/-10 \mathrm{~nm}$ [6]. The layout of a prototype two ${ }^{31} \mathrm{P}$ atom test structure is shown in an SEM image in Figure 1. Here, a pair of silicon nanowire single electron transistors (SET) is formed by electron beam lithography with a $100 \mathrm{keV}$ electron beam and an HSQ (hydrogen silsesquioxane) resist process. SETs are needed as sensitive electrometers for spin dependent charge transfer measurements [6]. Control of the spin state of individual electrons is crucial for testing of proposed ${ }^{31} \mathrm{P}$ qubit device structures. In order to access the physics of the ${ }^{31} \mathrm{P}$ qubit in an all silicon, scalable architecture, we use single ion implantation to implant single $\mathrm{P}$ ions aligned to the nanowire SETs. We now discuss several critical issues of basic highly charged ion solid interaction in the context of this development.

The first critical issue when using highly charged dopants, such as ${ }^{31} \mathrm{P}^{\mathrm{q}^{+}}$( $\mathrm{q}=12$ to 15), is whether the high charge state will lead to enhanced defect formation in the silicon matrix, and consequently modified thermal budget requirements for damage repair and dopant activation through annealing. Sporn et al., [7] have shown strong preferential oxygen desorption for SHCI impacts on $\mathrm{SiO}_{2}$ films. Charge relaxation times for SHCI are longer in dielectrics than in metals and semimetals due to the reduced availability of 
free electrons for screening of the transient hollow atom [8]. A $10 \mathrm{keV}^{31} \mathrm{P}$ ion travels only a few nm during charge relaxation, and the $\sim 1.5 \mathrm{~nm}$ thick native oxide on silicon absorbs most of the initially deposited potential energy $\left(9.3 \mathrm{keV}\right.$ for $\left.\mathrm{P}^{15+}\right)$. The impact of single $\mathrm{P}^{15+}$ ions leads to enhanced emission oxygen from the oxide surface. Following implantation, wafers are annealed for damage repair and dopant activation [5, 9]. We are currently characterizing electrical properties of low dose $\left(<1 \mathrm{E} 13 \mathrm{~cm}^{-2}\right)$, low energy $(5$ to $20 \mathrm{keV}$ ) highly charged P implants in silicon and SOI [9].

The second issue when implanting highly charged dopant ions is their range profile as compared to that of singly charged ions. Pre-equilibrium energy loss enhancements [8] will compress range profiles at a given kinetic energy. In Figure 2, we show magnetic sector SIMS depth profiles of low dose $\left(1 \mathrm{E} 12 \mathrm{~cm}^{-2}\right) \mathrm{P}^{12+}$ implants in silicon. The implant energies where 10 and $80 \mathrm{keV}$. A reduced ion range due to enhanced surface near stopping during charge relaxation requires very high resolution in depth profiling. For ${ }^{31} \mathrm{P}$, magnetic sector SIMS is required in order to resolve ${ }^{31} \mathrm{P}$ from ${ }^{30} \mathrm{SiH}$. Further, SIMS of top surface layers is hampered by beam-target equilibration effects, and transients in relative sensitivity factors due to the changing chemical environment from the native oxide layer to bulk silicon. A cap oxide could be deposited prior to analysis, but this leads to enhanced beam induced mixing. Experiments with thin dielectric films and higher charged heavy ions can reveal the extend of pre-equilibrium stopping enhancements during low energy ion implantation [10].

Single ion implantation becomes interesting when individual dopant ions can be placed into regions with $<10 \mathrm{~nm}$ diameters with high efficiency. The placement accuracy requirement is specific for the two qubit interaction scheme to be implemented $[6,11]$. 
Coupling by direct wave function overlap through exchange interaction requires qubit spacings of order 10 to $20 \mathrm{~nm}$ due to the Bohr radius of bound ${ }^{31} \mathrm{P}$ donor electrons in silicon. Coupling through spin coherent electron shuttling in a nuclear-electron spin encoding scheme relaxes the spacing requirement contingent to the magnitude of spin coherence lengths. Placement accuracy translates into placement resolution and alignment of the implant step. The placement resolution requires use of low energy ions, so that range straggling does not limit the placement accuracy. Further, diffusion during annealing has to be minimal [5]. We control the beam spot size on target through collimation of a focused beam in a small aperture. Very high alignment accuracy can be achieved by placing this aperture into the tip of an AFM [12]. A schematic of our single ion implanter approach is shown in Figure 3. Highly charged ions are extracted from an appropriate ion source (EBIT, or REBIT [13]), and reach the target station after momentum analysis in a double focusing analyzing magnet. The implant station is equipped with deflectors and electrostatic lenses that steer and focus the beam through the pre-collimator to the pierced AFM tip [14]. Figure 4 shows a tip with hollow pyramid into which a $100 \mathrm{~nm}$ hole was drilled directly with a $30 \mathrm{keV} \mathrm{Ga}^{+}$in an FEI Strata 235 dual beam FIB. Hole diameters much below $100 \mathrm{~nm}$ are difficult to achieve in direct FIB drilling. In order to achieve the required placement accuracy, the spot size-limiting aperture needs to have a diameter $<10 \mathrm{~nm}$. High aspect ratio ( $>4: 1)$ holes with sub $10 \mathrm{~nm}$ diameters can be formed by thin film deposition over a larger hole [15]. In Figure 5, we show a scanning transmission electron microscope line scan of Pt x-rays across the hole shown in the insert. Here, a larger hole ( $\sim 100 \mathrm{~nm}$ diameter $)$ had been drilled directly with the FIB beam into a silicon nitride membrane ( $200 \mathrm{~nm}$ thick). We then deposited a 
Pt film of several hundred nm thickness in the area of the primary hole by standard ion beam assisted thin film deposition. During thin film deposition, the hole closes and the hole diameter is reduced down to a value of about $5 \mathrm{~nm}$.

It is now of great interest to see how such small apertures respond to exposure to SHCI and highly charged dopant ions. Clearly, charge state enhanced electronic sputtering will reduce mask lifetimes. However, electronic sputtering has only been observed for dielectric films and some semiconductors, not for metal, and a metal coating can be applied to suppress electronic sputtering. A subtlety here is the recently reported effect of hole closing during beam exposure for $\mathrm{Ar}^{1+}$ ions. The balance of ad-atom formation and diffusion and material removal by sputtering can be adjusted with the target temperature and dose rate to open or close small holes [16]. It can be expected that the optimal conditions for steady hole diameters are charge state dependent. Besides electronic sputtering, charge exchange is on important process in the interaction of SHCI with small apertures $[17,18]$. Transport of ions through nanometer (few hundred nanometer diameters) scale dielectric apertures has recently been reported by Stolterfoht et al. [18]. Here, charge exchange was found to be suppressed by channel wall charging even when collisions with capillary walls were forced by foil tilting. In Figure 6, we show first data of charge state distributions for $\mathrm{Ar}^{3+}$ ion $\left(\mathrm{E}_{\mathrm{kin}}=9.6 \mathrm{keV}\right)$ transmitted through $30 \mathrm{~nm}$ wide holes in a $130 \mathrm{~nm}$ thick nickel membrane on a silicon frame. A classical over the barrier model [19] predicts a charge exchange fraction, $f$, given by the ratio of the critical radius for resonant electron capture from the channel walls, $\mathrm{d}$, to the channel diameter, $\mathrm{r}, \mathrm{f} \sim 2 \mathrm{~d} / \mathrm{r}$. The data for the rather low charge state $\mathrm{Ar}^{3+}$ show a charge exchange fraction of $<1 \%$ and are, as expected, consistent with this 
prediction. The critical radius for resonant electron capture form a $5 \mathrm{eV}$ work function metal surface into a $\mathrm{Au}^{69+}$ ion is about $5 \mathrm{~nm}$. It will be very interesting to see charge exchange data for sub-10 nm capillaries and very highly charged ions, where critical radii for electron capture can approach channel diameters.

\section{Summary}

In this article we discuss open questions related to electronic sputtering of solids by slow, highly charged ions in the context of our development of a single ion implantation scheme with highly charged dopant ions. Results from fundamental studies in recent years provide important background data, and point to experiments needed in order to quantify charge state effects on electrical properties of silicon implanted with highly charged dopant ions, electronic sputtering effect on beam collimator lifetimes, and charge exchange processes in nanometer scale capillaries.

\section{Acknowledgments}

This work was performed at the National Center for Electron Microscopy at the E. O. Lawrence Berkeley National Laboratory and was supported by the National Security Agency and Advanced Research and Development Activity under Army Research Office contract number MOD707501, and by the U. S. Department of Energy under contract No. DE-AC03-76SF00098.

Permanent addresses:

*Institute of Microstructure Technologies and Analytics (IMA), University of Kassel, 
Heinrich Plett Straße 40, 34132 Kassel, Germany

**Institute of Electron Technology, Al. Lotion 11, 02-688 Warszawa, Poland

\section{References}

[1] T. Schenkel et al., Prog. Surf. Sci. 61, 23 (1999)

[2] A. Arnau, et al., Surf. Sci. Rep. 27, 117 (1997)

[3] HP. Winter, and F. Aumayr, Phys. Scr. T92, 12 (2001)

[4] T. Schenkel, et al., Nucl. Instr. Meth. B 161-163, 65 (2000)

[5] T. Schenkel, et al., J. Vac. Sci. Technol. B 20, 2819 (2002)

[6] B. E. Kane, Nature 393, 133 (1998); A. J. Skinner, M. E. Davenport, B. E. Kane, Phys. Rev. Lett. 90, 087901 (2003)

[7] M. Sporn, et al., Phys. Rev. Lett. 79, 945 (1997)

[8] T. Schenkel, et al., Phys. Scr. T92, 208 (2001); Phys. Rev. Lett. 79, 2030 (1997)

[9] T. Schenkel, proceedings of the $5^{\text {th }}$ Workshop on Ultrashallow Junction Formation, Santa Cruz, CA, April 2003, to be published in J. Vac. Sci. Technol. B

[10] M. A. Briere, J. P. Biersack, and D. H. Schneider, LLNL-EBIT, Annual Report, 1993, UCRL-ID-118274 (unpublished)P. 33; J. P. Biersack, Nucl. Instr. Meth. B $80 / 81,12(1993)$

[11] M. A. Nielson, I. L. Chuang, Quantum Computation (University Press, Cambridge, 2000); see also the recently published roadmap for quantum information science and technology under: http://qist.lanl.gov/.

[12] I. W. Rangelow, J. Voigt, and K. Edinger, J. Vac. Sci. Technol. B16, 2723 (2001); R. Lüthi, et al., Appl. Phys. Lett. 75, 1314 (1999); R. G. Clark, unpublished 
[13] T. Schenkel, et al., Rev. Sci. Instr. 73, 663 (2002)

[14] I. W. Rangelow, et al., Proceedings of the $47^{\text {th }}$ International Conference on Electron, Ion and Photon Beam Technology and Nanofabrication, Tampa, FL, May 2003, to be published in J. Vac. Sci. Technol. B

[15] T. Schenkel, et al., Proceedings of the $47^{\text {th }}$ International Conference on Electron, Ion and Photon Beam Technology and Nanofabrication, Tampa, FL, May 2003, to be published in J. Vac. Sci. Technol. B

[16] J. Li, D. Stein, C. McMullan, D. Branton, M. J. Aziz, and J. A. Golovchenko, Nature 412, $166(2001)$

[17] S. Ninomiya, et al., Phys. Rev. Lett 78, 4557 (1997)

[18] N. Stolterfoht, et al., Phys. Rev. Lett. 88, 133201 (2002)

[19] J. Burgdörfer, P. Lerner, and F. W. Meyer, Phys. Rev. A 44, 5647 (1991) 


\section{Figure captions:}

Figure 1: Layout of a pair of silicon nanowire single electron transistors in SOI with positions of ${ }^{31} \mathrm{P}$ atoms indicated for spin dependent charge measurements. The width of the silicon wire is $10.5 \mathrm{~nm}$.

Figure 2: Magnetic sector SIMS depth profiles of ${ }^{31} \mathrm{P}$ atoms in silicon wafers from implantation of $\mathrm{P}^{12+}$ ions with kinetic energies of $10 \mathrm{keV}$ (dashed) and $80 \mathrm{keV}$ (solid).

Figure 3: Schematic of the Single Ion Implantation setup with AFM alignment. 1: piezoresisitive AFM cantilever with hollow tip and small aperture (2), 3: high resolution sample stage, and secondary electron detector (4), 5: pre-collimator.

Figure 4: Piezo-resistive AFM tip (left, A) with pierced, hollow metal pyramid (right, B). The hole diameter is $100 \mathrm{~nm}$ (bottom, C).

Figure 5: STEM line scan of Pt x-ray intensities across a FIB drilled $100 \mathrm{~nm}$ wide hole following in situ ion beam assisted Pt deposition. The residual hole diameter is about 5 nm. The substrate was a $200 \mathrm{~nm}$ thick, low stress silicon nitride membrane.

Figure 6: Charge state distribution following transmission of $\mathrm{Ar}^{3+}$ ions $\left(\mathrm{E}_{\mathrm{kin}}=9.6 \mathrm{keV}\right)$ through $30 \mathrm{~nm}$ wide holes in a $130 \mathrm{~nm}$ thick Ni membrane. An SEM image of the hole area on the membrane is shown in the insert. 


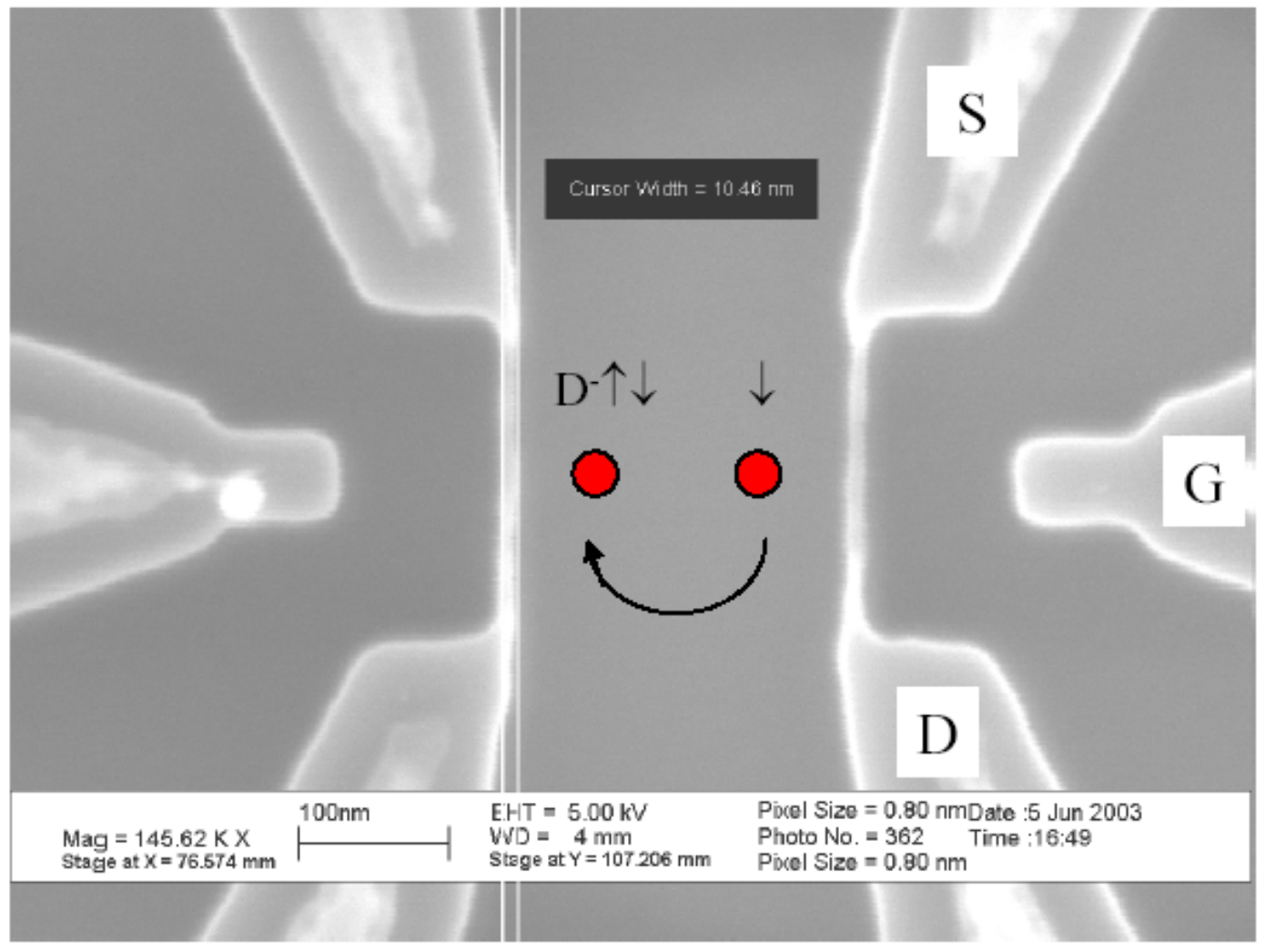




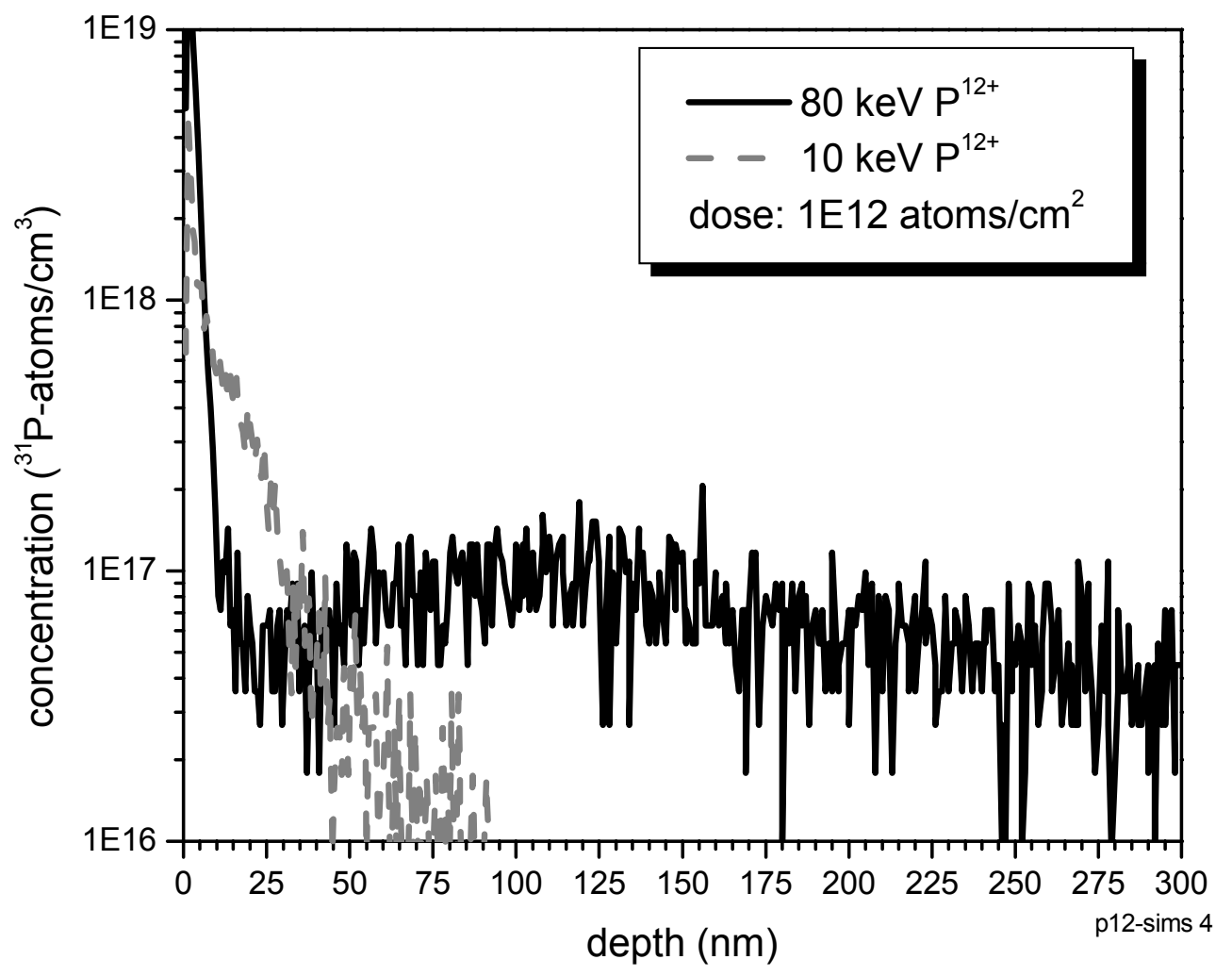




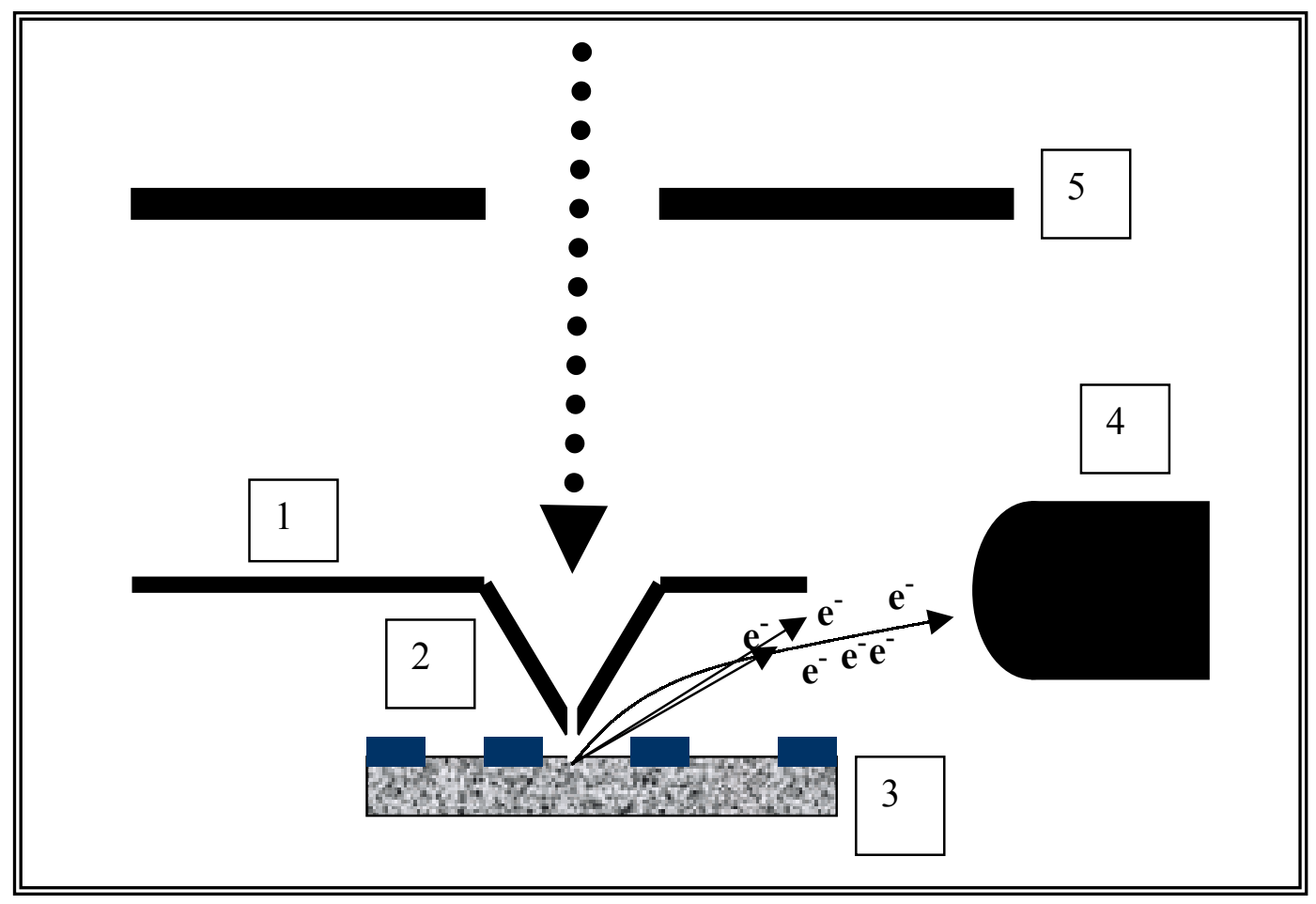



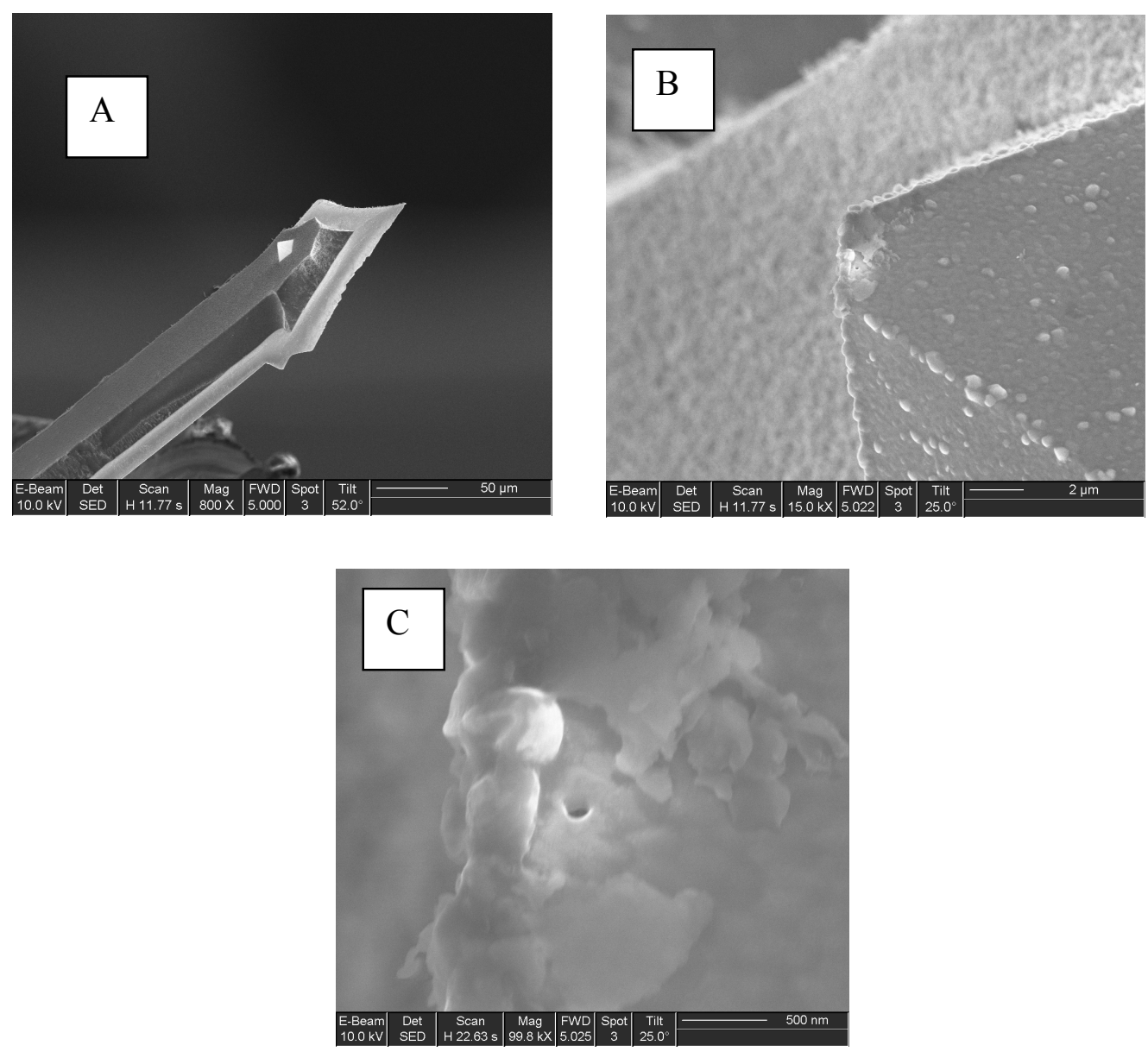


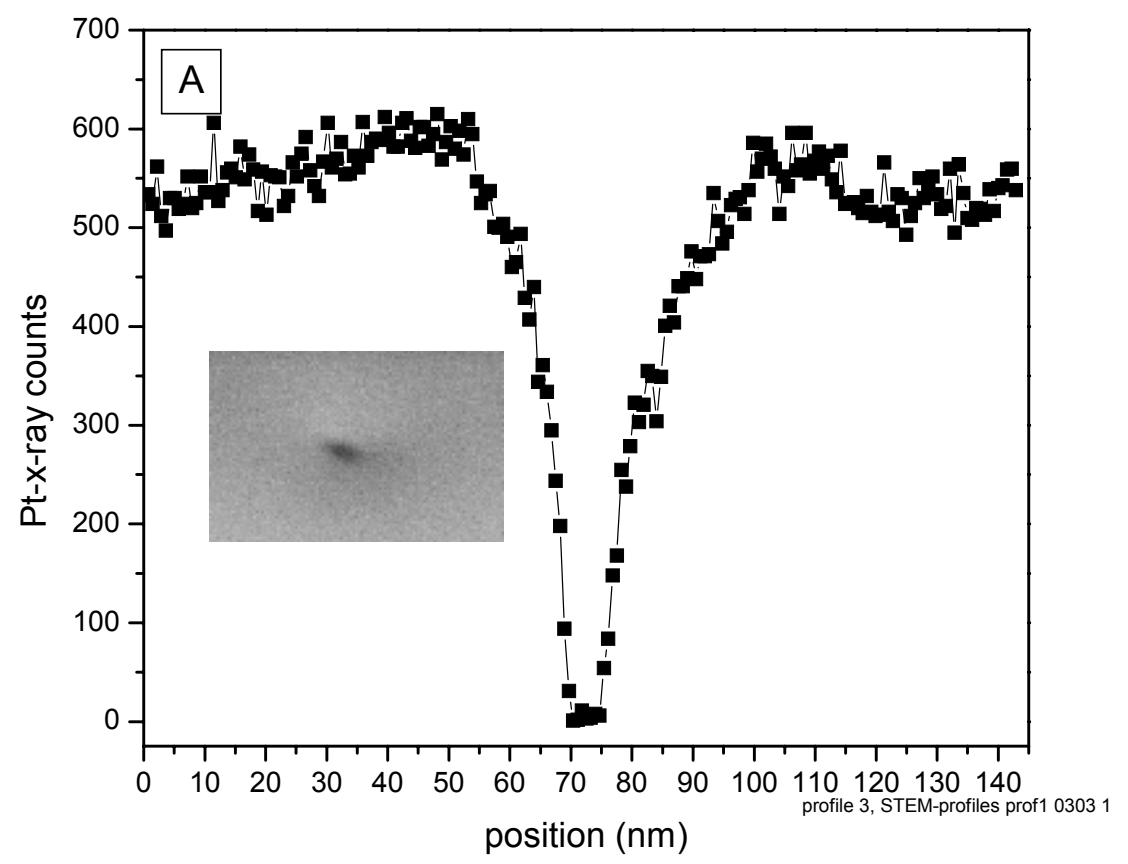




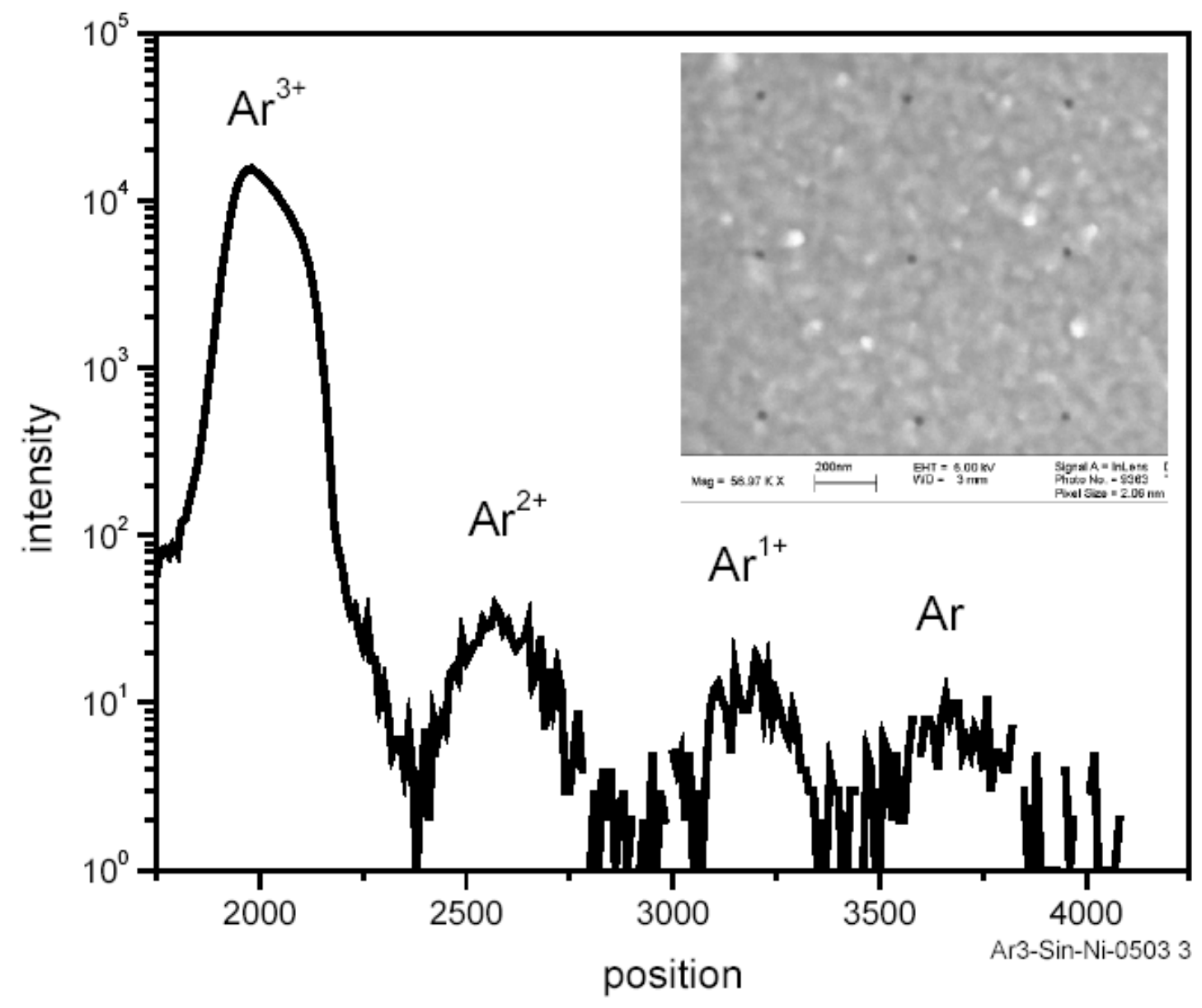

